\title{
Multivariate Markov Families of Copulas
}

DOI 10.1515/demo-2015-0011

Received July 8, 2015; accepted October 9, 2015

\begin{abstract}
For the Markov property of a multivariate process, a necessary and sufficient condition on the multidimensional copula of the finite-dimensional distributions is given. This establishes that the Markov property is solely a property of the copula, i.e., of the dependence structure. This extends results by Darsow et al. [11] from dimension one to the multivariate case. In addition to the one-dimensional case also the spatial copula between the different dimensions has to be taken into account. Examples are also given.
\end{abstract}

Keywords: Markov process; copula; Chapman-Kolmogorov equation

MSC: Primary 60G07, secondary 60J05, 60J25

\section{Introduction}

We study the Markov property of multi-dimensional stochastic processes in terms of the corresponding multidimensional copulas. Let $\mathbf{X}=\left(\mathbf{X}_{t}\right)_{t \in \mathcal{T}}$ with $\mathcal{T} \subseteq \mathbb{R}^{+}$be a $n$-dimensional stochastic process with coordinates $X^{i}=\left(X_{t}^{i}\right)_{t \in \mathcal{T}}, i=1, \ldots, n$, defined on some filtered probability space $\left(\Omega, \mathcal{F}, \mathbb{F}=\left(\mathcal{F}_{t}\right)_{t \in \mathcal{T}}, \mathbf{P}\right)$. We study properties of the copulas between random variables $X_{t}^{i}, X_{s}^{j}, i, j=1, \ldots, n$, that guarantee the Markov property of the process $\mathbf{X}$. For the Chapman-Kolmogorov equation to hold true, we give a necessary and sufficient condition on the involved copulas. Based on that we provide a necessary and sufficient condition for $\mathbf{X}$ to be a Markov process. For the process $\mathbf{X}$ to be Markov, the one-dimensional distributions of its coordinates $X_{t}^{i}$ do not matter. The Markov property is a pure "dependence" property that can be formulated exclusively in terms of copulas. As a consequence, starting with a Markov process $\mathbf{X}$, a multitude of other Markov processes can be constructed by just modifying the marginal distributions.

So far copula based approaches to Markov processes mainly focus on one-dimensional processes; we refer to the grounding paper by Darsow et al. [11], but also to [13], [1], [7], [8], and [2]. Our paper generalizes results derived by Darsow et al. [11], for the one-dimensional case to the general multivariate setting. Here we have to take into account that the $n$-dimensional marginal distribution of $\mathbf{X}_{t}$ admits a spatial copula, whereas in the one-dimensional case marginal distributions can be simply transformed into a uniform distribution. Understanding the interplay between the multivariate spatial copulas over time is the main challenge that is met by an appropriate generalization of the ${ }^{\star}$-operator from [11].

Recent results on estimation and modeling based on multivariate time series with focus on copulas can be found e.g. in [17], [18], [20], [19], and [3]. The latter one considers vine copulas, which are related to pair copulas, as e.g. in [12], and [6]. The work in [3] considers also the question, when does a vine copula specification related to a time series give rise to a Markov process. Vine copulas are specified by conditional bivariate copulas. However their results do not carry over to a continuous time setting in a straightforward way.

In [13] the main result from [11] is extended in discrete time to processes with the $k$-period Markov property; here the last $k$ time points are a sufficient statistics for the distribution of the following time point. In

^Corresponding Author: Wolfgang M. Schmidt: Frankfurt School of Finance and Management, Sonnemannstr. 9-11, 60314 Frankfurt am Main, E-mail: w.schmidt@fs.de

Ludger Overbeck: Justus-Liebig Universität Gießen, Institut of Mathematics, 35392 Gießen 
addition it is assumed that the time series is stationary, i.e., all $k$-time points have the same $k$-dimensional copula.

The transition function of a Markov chain can be written in terms of the copula of the joint distribution of two consecutive time points, a fact that is used in [1] for maximum likelihood estimation.

The concept of copula had recently a revival in particular motivated by its application quantitative risk management and mathematical finance, see e.g., [15]. [9], [10] and [4] investigate the dependence structure of stochastic processes with copula-like methods in the context of applications in mathematical finance.

The paper [5] considers a multivariate process with given infinitesimal characteristics, whose onedimensional components coincide in law with some given one-dimensional processes. For a diffusion this requires a relation between the diagonal and the off-diagonal entries of the (instantaneous) covariance/diffusion matrix. That differs basically from our approach, which relies solely on the specification and analysis of copulas of finite-dimensional distributions.

The paper is organized as follows. In Section 2 we define a product operator between copulas and study properties of this operator that are essential for formulating our results. Multivariate Markov processes and their copulas are studied in Section 3. Finally, in Section 4 as an example we propose a family of Fréchet type copulas that lead to a class of Markov processes and calculate their associated infinitesimal generators.

\section{Notation and auxiliary results}

Let $\mathbf{X}=\left(X^{1}, \ldots, X^{k}\right), \mathbf{Y}=\left(Y^{1}, \ldots, Y^{l}\right)$ be $k$ and $l$-dimensional random variables and denote by $F_{X^{i}}, F_{Y^{i}}$ the marginal distribution function of $X^{i}$ and $Y^{i}$, respectively. A copula $C$ of a random vector $\left(X^{1}, \ldots, X^{k}\right)$ is a distribution function $C$ on $[0,1]^{k}$ such that the joint distribution $F_{\mathbf{X}}$ of the vector $\mathbf{X}=\left(X^{1}, \ldots, X^{k}\right)$ satisfies

$$
F_{\mathbf{X}}\left(x_{1}, ., x_{k}\right)=C\left(F_{X_{1}}\left(x_{1}\right), ., F_{X_{k}}\left(x_{k}\right)\right) .
$$

We refer to [16], [14] for exhaustive presentations of the concept of copulas.

The copula of the vector $\left(X^{1}, \ldots, X^{k}, Y^{1}, \ldots, Y^{l}\right)$ is denoted by $C\left(x^{1}, \ldots, x^{k}, y^{1}, \ldots, y^{l}\right)$. We follow [11] and assume a coordinate-wise linear interpolation for those points where the copula is not uniquely defined. To lighten notation we frequently use the following short hand notation,

$$
\begin{aligned}
\mathbf{x} & =x^{1}, \ldots, x^{k} \\
\mathrm{~d} \mathbf{x} & =\mathrm{d} x^{1} \ldots \mathrm{d} x^{k} \\
\mathbf{F}_{\mathbf{X}}(\mathbf{x}) & =F_{X^{1}}\left(x^{1}\right), \ldots, F_{X^{k}}\left(x^{k}\right) \\
\mathbf{X}<\mathbf{x} & =X^{1}<x^{1}, \ldots, X^{k}<x^{k},
\end{aligned}
$$

and accordingly for $\mathbf{y}, \mathbf{d} \mathbf{y}, \mathbf{F}_{\mathbf{Y}}(\mathbf{y}), \mathbf{Y}<\mathbf{y}$, etc. Observe the difference in the notations $F_{\mathbf{X}}$ and $\mathbf{F}_{\mathbf{X}}$.

The copula of the vector $\mathbf{Y}$ is denoted by $C^{\mathbf{Y}}(\mathbf{y})$. We define the derivative of the copula $C$ with respect to another copula $A$, in particular $A=C^{\mathbf{Y}}$. Recall that, for every fixed vector $\mathbf{x}$ the function $C(\mathbf{x},$.$) is the$ distribution function of a measure on $[0,1]^{l}$.

Definition 1. Assume that, for $\mathbf{x} \in[0,1]^{k}$ the distribution $C(\mathbf{x},$.$) is absolutely continuous with respect to the$ measure generated by some copula $A$ on $[0,1]^{l}$. We denote by $C_{, A}(\mathbf{x}, \mathbf{y})$ (a version of) the Radon-Nikodym derivative,

$$
C(\mathbf{x}, \mathrm{d} \mathbf{y})=C_{, A}(\mathbf{x}, \mathbf{y}) A(\mathrm{~d} \mathbf{y}) .
$$

The subscript ", $A$ " indicates that we take the derivative with respect to the second set of arguments $(\mathbf{y})$. Accordingly, we define the derivative $C_{B},(\mathbf{x}, \mathbf{y})$ of $C$ with respect to a $k$-dimensional copula $B$ by

$$
C(\mathrm{~d} \mathbf{x}, \mathbf{y})=C_{B},(\mathbf{x}, \mathbf{y}) B(\mathrm{~d} \mathbf{x}),
$$


provided that for given $\mathbf{y}$ the measure generated by $C(., \mathbf{y})$ is absolutely continuous with respect to the measure generated by $B . C_{, A}(\mathbf{x}, \mathbf{y})$ and $C_{B},(\mathbf{x}, \mathbf{y})$ are called derivative of the copula $C(\mathbf{x},$.$) resp. C(., \mathbf{y})$ with respect to the copula A resp. $B$.

The derivatives $C_{, C^{\mathrm{y}}}$ and $C_{C^{\mathrm{x}}}$, are clearly well-defined for all $\mathbf{x}$ and all $\mathbf{y}$.

The derivative $C_{, A}(\mathbf{x}, \mathbf{y})$ is increasing in $\mathbf{x}$, more precisely, for $\mathbf{v} \geq \mathbf{u}$ we have

$$
C_{, A}(\mathbf{v}, \mathbf{y})-C_{, A}(\mathbf{u}, \mathbf{y}) \geq 0,
$$

for $A$-almost all $\mathbf{y}$.

Lemma 1. We have the following expressions for conditional distributions

$$
\begin{aligned}
& \mathbf{P}(\mathbf{X}<\mathbf{x} \mid \mathbf{Y})(\omega)=C_{C^{\mathbf{Y}}}\left(\mathbf{F}_{\mathbf{X}}(\mathbf{x}), \mathbf{F}_{\mathbf{Y}}(\mathbf{Y}(\omega))\right), \mathbf{P}-\text { a.s. } \\
& \mathbf{P}(\mathbf{Y}<\mathbf{y} \mid \mathbf{X})(\omega)=C_{C^{\mathrm{X}},}\left(\mathbf{F}_{\mathbf{X}}(\mathbf{X}(\omega)), \mathbf{F}_{\mathbf{Y}}(\mathbf{y})\right), \mathbf{P}-\text { a.s. }
\end{aligned}
$$

Proof. To prove (2), we have to show that for every $\mathbf{a}=\left(a^{1}, \ldots, a^{l}\right)$

$$
\mathbf{P}(\mathbf{X}<\mathbf{x}, \mathbf{Y}<\mathbf{a})=\int_{\{\omega: \mathbf{Y}(\omega)<\mathbf{a}\}} C_{, C^{\mathbf{Y}}}\left(\mathbf{F}_{\mathbf{X}}(\mathbf{x}), \mathbf{F}_{\mathbf{Y}}(\mathbf{Y}(\omega))\right) \mathbf{P}(\mathrm{d} \omega) .
$$

The right hand side can be rewritten as

$$
\begin{aligned}
\int_{\{\omega: \mathbf{Y}(\omega)<\mathbf{a}\}} C_{, C^{\mathbf{Y}}}\left(\mathbf{F}_{\mathbf{X}}(\mathbf{x}), \mathbf{F}_{\mathbf{Y}}(\mathbf{Y}(\omega))\right) \mathbf{P}(\mathrm{d} \omega) & =\int_{\mathbf{y}<\mathbf{a}} C_{, C^{\mathbf{Y}}}\left(\mathbf{F}_{\mathbf{X}}(\mathbf{x}), \mathbf{F}_{\mathbf{Y}}(\mathbf{y})\right) \mathrm{d} F_{\mathbf{Y}}(\mathbf{y}) \\
& =\int_{-\infty}^{a^{1}} \ldots \int_{-\infty}^{a^{l}} C_{, C^{\mathbf{Y}}}\left(\mathbf{F}_{\mathbf{X}}(\mathbf{x}), \mathbf{F}_{\mathbf{Y}}(\mathbf{y})\right) C^{\mathbf{Y}}\left(F_{Y^{1}}\left(\mathrm{~d} y^{1}\right), \ldots, F_{Y^{l}}\left(\mathrm{~d} y^{l}\right)\right) .
\end{aligned}
$$

By applying the change of variables rule to each integral we arrive at

$$
\int_{0}^{F_{Y^{1}}\left(a^{1}\right)} \ldots \int_{0}^{F_{Y^{l}}\left(a^{l}\right)} C_{, C^{\mathbf{Y}}}\left(\mathbf{F}_{\mathbf{X}}(\mathbf{x}), F_{Y^{i}}\left(F_{Y^{i}}^{-1}\left(z^{i}\right)\right), i=1, \ldots, l\right) C^{\mathbf{Y}}\left(\mathrm{d} z^{1}, \ldots, \mathrm{d} z^{l}\right),
$$

where $F_{Y^{i}}^{-1}(z)=\inf \left\{t \geq 0: F_{Y^{i}}(t)>z\right\}$ is the right-inverse of $F_{Y^{i}}$. If the marginal distributions $F_{Y^{i}}$ are continuous, then we have $F_{Y^{i}}\left(F_{Y^{i}}^{-1}\left(z^{i}\right)\right)=z^{i}$ and we can continue by writing

$$
\begin{aligned}
& C\left(\mathbf{F}_{\mathbf{X}}(\mathbf{x}), \mathbf{F}_{\mathbf{Y}}(\mathbf{y})\right)= \\
& \quad \int_{0}^{F_{Y^{1}}\left(a^{1}\right)} \ldots \int_{0}^{F_{Y^{l}}\left(a^{l}\right)} C_{, C^{\mathbf{Y}}}\left(\mathbf{F}_{\mathbf{X}}(\mathbf{x}), z^{1}, \ldots, z^{l}\right) C^{\mathbf{Y}}\left(\mathrm{d} z^{1}, \ldots, \mathrm{d} z^{l}\right),
\end{aligned}
$$

which finishes the proof in this case. For the general case, if $y^{i}$ is a point of discontinuity of $F_{Y^{i}}$, with $F_{Y^{i}}\left(y^{i}-\right)=$ $u^{i}, F_{Y^{i}}\left(y^{i}+\right)=v^{i}$, then the copulas $C$ and $C^{\mathbf{Y}}$ are not uniquely determined in the interval $\left(u^{i}, v^{i}\right)$ for the $i$-th coordinate of $\mathbf{Y}$. Moreover, $F_{Y^{i}}\left(F_{Y^{i}}^{-1}\left(z^{i}\right)\right)=v^{i}$ for all $z^{i} \in\left(u^{i}, v^{i}\right)$. Then (4) still reduces to $C\left(\mathbf{F}_{\mathbf{X}}(\mathbf{x}), \mathbf{F}_{\mathbf{Y}}(\mathbf{y})\right)$, if

$$
C_{, C^{\mathbf{Y}}}\left(\mathbf{F}_{\mathbf{X}}(\mathbf{x}), \ldots, v^{i}, \ldots\right)=\frac{C\left(\mathbf{F}_{\mathbf{X}}(\mathbf{x}), \ldots, v^{i}, \ldots\right)-C\left(\mathbf{F}_{\mathbf{X}}(\mathbf{x}), \ldots, u^{i}, \ldots\right)}{C^{\mathbf{Y}}\left(\ldots, v^{i}, \ldots\right)-C^{\mathbf{Y}}\left(\ldots, u^{i}, \ldots\right)},
$$

which holds because of our assumption that copulas will be coordinate-wise linearly interpolated for all intervals where they are not unique.

The proof of (3) is analogously. 
Remark 1. If the copula $C(\mathbf{x}, \mathbf{y})$ has a density $c$, i.e.,

$$
C(\mathbf{x}, \mathbf{y})=\int_{\mathbf{0}}^{\mathbf{x}} \int_{\mathbf{0}}^{\mathbf{y}} c(\mathbf{u}, \mathbf{v}) \mathrm{d} \mathbf{u} \mathrm{d} \mathbf{v},
$$

then $C_{{ }_{C^{Y}}}$ coincides with the familiar conditional density,

$$
C_{, C^{Y}}(\mathbf{x}, \mathbf{z})=\frac{\int_{\mathbf{0}}^{\mathbf{x}} c(\mathbf{u}, \mathbf{z}) \mathrm{d} \mathbf{u}}{\int_{\mathbf{0}}^{\mathbf{1}} c(\mathbf{u}, \mathbf{z}) \mathrm{d} \mathbf{u}}
$$

Definition 2 (The $\stackrel{C(.)}{\star}$ product operator). Let $A$ be $a(k+m)$-dimensional copula, $B$ be $a(m+l)$-dimensional copula and $C$ be a m-dimensional copula such that the derivatives $A_{, C}$ and $B_{C}$, are well-defined. The operator $\stackrel{C(.)}{\star}$ is defined by

$$
(A \stackrel{C(\mathbf{z})}{\star} B)(\mathbf{x}, \mathbf{y})=\int_{\mathbf{0}}^{\mathbf{z}} A_{, C}(\mathbf{x}, \mathbf{r}) \cdot B_{C},(\mathbf{r}, \mathbf{y}) C(\mathrm{~d} \mathbf{r}),
$$

provided that the integral exists for all $\mathbf{x}, \mathbf{y}, \mathbf{z}$. To shorten notation, we denote $\left(A^{\star}{ }^{C} B\right)(\mathbf{x}, \mathbf{y})=(A \stackrel{C(\mathbf{1})}{\star} B)(\mathbf{x}, \mathbf{y})$.

In case $k=l=m=1$ the operator ${ }^{\star}$ r reduces to the ${ }^{\star}$ operator that was introduced by Darsow et al. [11]: for two-dimensional copulas $A(x, y), B(x, y)$,

$$
(A \star B)(x, y)=\int_{0}^{1} A_{, z}(x, z) \cdot B_{z},(z, y) \mathrm{d} z,
$$

with $A_{, z}(x, z)$ the derivative of $A$ with respect to the second argument, and accordingly for $B_{z},(z, y)$. The key for extending the results of [11] to the multivariate case is the notion of the derivative of a copula with respect to another copula in Definition 1, which is the basis for the definition of the ${ }^{\star}$ operator.

In our applications of the ${ }^{C(.)}$ product operator in relation to Markov processes, we encounter the following situation in which the $\stackrel{C(.)}{\star}$ product is always well-defined.

Lemma 2. Consider random vectors $\mathbf{X}, \mathbf{Z}, \mathbf{Y}$ with dimensions $k, m, l$, respectively, and let $A$ (resp. B) denote the copula between $\mathbf{X}, \mathbf{Z}$ (resp. Z, Y),

$$
\begin{aligned}
& \mathbf{P}(\mathbf{X}<\mathbf{x}, \mathbf{Z}<\mathbf{z})=A\left(\mathbf{F}_{\mathbf{X}}(\mathbf{x}), \mathbf{F}_{\mathbf{Z}}(\mathbf{z})\right), \\
& \mathbf{P}(\mathbf{Z}<\mathbf{z}, \mathbf{Y}<\mathbf{y})=B\left(\mathbf{F}_{\mathbf{Z}}(\mathbf{z}), \mathbf{F}_{\mathbf{Y}}(\mathbf{y})\right) .
\end{aligned}
$$

For $C$ denoting the copula of $\mathbf{Z}$, the operator

$$
(A \stackrel{C(\mathbf{z})}{\star} B)(\mathbf{x}, \mathbf{y})=\int_{\mathbf{0}}^{\mathbf{z}} A_{, C}(\mathbf{x}, \mathbf{r}) \cdot B_{C},(\mathbf{r}, \mathbf{y}) C(\mathrm{~d} \mathbf{r}),
$$

is well-defined.

Proof. For every $\mathbf{x}$ and every Borel set $z \in \mathfrak{B}\left([0,1]^{m}\right)$ we have

$$
\int_{Z} A_{, C}(\mathbf{x}, \mathbf{r}) C(\mathrm{~d} \mathbf{r})=\int_{Z} A(\mathbf{x}, \mathrm{d} \mathbf{r}) \leq \int_{Z} A(\mathbf{1}, \mathrm{d} \mathbf{r})=\int_{Z} C(\mathrm{~d} \mathbf{r}) .
$$

This implies $A_{, C}(\mathbf{x}, \mathbf{r}) \leq 1$ for $C$-almost all r. Accordingly, $B_{C},(\mathbf{r}, \mathbf{y}) \leq 1$ for $C$-almost all $\mathbf{r}$. Thus the integrand in (8) is bounded $C$-almost surely and the assertion follows.

The following Lemma illustrates the role played by the $\stackrel{C(.)}{\star}$ operator. 
Lemma 3. Consider random vectors $\mathbf{X}, \mathbf{Z}, \mathbf{Y}$ with dimensions $k, m, l$, respectively, and let $A$ (resp. $B$ ) denote the copula between $\mathbf{X}, \mathbf{Z}$ (resp. Z, Y),

$$
\begin{aligned}
& \mathbf{P}(\mathbf{X}<\mathbf{X}, \mathbf{Z}<\mathbf{z})=A\left(\mathbf{F}_{\mathbf{X}}(\mathbf{x}), \mathbf{F}_{\mathbf{Z}}(\mathbf{z})\right), \\
& \mathbf{P}(\mathbf{Z}<\mathbf{z}, \mathbf{Y}<\mathbf{y})=B\left(\mathbf{F}_{\mathbf{Z}}(\mathbf{z}), \mathbf{F}_{\mathbf{Y}}(\mathbf{y})\right) .
\end{aligned}
$$

Let $C$ denote the copula of $\mathbf{Z}$. Then $\mathbf{X}, \mathbf{Y}$ are conditionally independent given $\mathbf{Z}$ if and only if

$$
\mathbf{P}(\mathbf{X}<\mathbf{x}, \mathbf{Y}<\mathbf{y}, \mathbf{Z}<\mathbf{z})=\left(A \stackrel{C\left(\mathbf{F}_{\mathbf{Z}}(\mathbf{z})\right)}{\star} B\right)\left(\mathbf{F}_{\mathbf{X}}(\mathbf{x}), \mathbf{F}_{\mathbf{Y}}(\mathbf{y})\right) .
$$

Proof. $\mathbf{X}, \mathbf{Y}$ are conditionally independent given $\mathbf{Z}$ if and only if

$$
\begin{aligned}
\mathbf{P}(\mathbf{X}<\mathbf{X}, \mathbf{Y}<\mathbf{y}, \mathbf{Z}<\mathbf{z}) \\
\quad=\int_{\{\omega: \mathbf{Z}(\omega)<\mathbf{z}\}} \mathbf{P}(\mathbf{X}<\mathbf{X} \mid \sigma(\mathbf{Z}))(\omega) \mathbf{P}(\mathbf{Y}<\mathbf{y} \mid \sigma(\mathbf{Z}))(\omega) \mathbf{P}(\mathrm{d} \omega) .
\end{aligned}
$$

By using Lemma (1) the assertion follows.

Proposition 1. $(A \stackrel{C(\mathbf{z})}{\star} B)(\mathbf{x}, \mathbf{y})$ is $a(k+m+l)$-dimensional copula. In particular, $\left(A{ }^{\star} B\right)(\mathbf{x}, \mathbf{y})$ is $a(k+l)$ dimensional copula.

Proof. By Lemma (1), $A_{, C}$ and $B_{C}$, are Markov kernels, i.e. for every $\mathbf{z}, A_{, C}(., \mathbf{z})$ defines a probability measure on $[0,1]^{k}$ and $A_{, C}(X, \mathbf{z})$ is measurable in $\mathbf{z}$ for every $X \in \mathfrak{B}\left([0,1]^{k}\right)$, and correspondingly for $B_{C}$, Then the product $A_{, C}(., \mathbf{z}) B_{C},(\mathbf{z},$.$) generates a (product) probability measure on [0,1]^{k+l}$ that is again measurable in z. Finally, integrating w.r.t. $\mathbf{z}^{1}$,

$$
\int_{Z} A_{, C}(X, \mathbf{z}) \cdot B_{C},(\mathbf{z}, y) C(\mathrm{~d} \mathbf{z}), X \in \mathfrak{B}\left([0,1]^{k}\right), y \in \mathfrak{B}\left([0,1]^{l}\right), z \in \mathfrak{B}\left([0,1]^{m}\right)
$$

generates a probability measure on $[0,1]^{k+m+l}$ whose distribution function is just $(A \stackrel{C(\mathbf{z})}{\star} B)(\mathbf{x}, \mathbf{y})$. Since the marginals of this distribution are uniform, the assertion follows.

The following lemma will be crucial for our copula analysis for Markov processes.

Lemma 4. Let $A, B, C, D, E$ be copulas with dimensions $(k+m),(m+l), m, k, l$, respectively. Assume the ${ }^{C(.)} \star$ product and the derivatives w.r.t. $D$ (resp. E) below are all well-defined. Then

$$
\begin{aligned}
& (A \stackrel{C(\mathbf{z})}{\star} B)_{D},(\mathbf{x}, \mathbf{y})=\int_{\mathbf{0}}^{\mathbf{z}} B_{C},(\mathbf{r}, \mathbf{y}) \cdot A_{D},(\mathbf{x}, \mathrm{d} \mathbf{r}) \\
& (A \stackrel{\mathrm{Z}(\mathbf{z})}{\star} B)_{, E}(\mathbf{x}, \mathbf{y})=\int_{\mathbf{0}}^{\mathbf{z}} A_{, C}(\mathbf{x}, \mathbf{r}) \cdot B_{, E}(\mathrm{~d} \mathbf{r}, \mathbf{y}) .
\end{aligned}
$$

Proof. By definition of the derivative as Radon-Nikodym density,

$$
(A \stackrel{C(\mathbf{z})}{\star} B)(\mathbf{x}, \mathbf{y})=\int_{\mathbf{0}}^{\mathbf{x}}(A \stackrel{C(\mathbf{z})}{\star} B)_{D},(\mathbf{v}, \mathbf{y}) D(\mathrm{~d} \mathbf{v})
$$

1 Observe that the integral is well-defined by definition of the $\stackrel{C(.)}{\star}$ operator. 
On the other hand, by applying Fubini's theorem,

$$
\begin{aligned}
(A \stackrel{C(\mathbf{z})}{*} B)(\mathbf{x}, \mathbf{y}) & =\int_{\mathbf{0}}^{\mathbf{z}} A_{, C}(\mathbf{x}, \mathbf{r}) \cdot B_{C},(\mathbf{r}, \mathbf{y}) C(\mathrm{~d} \mathbf{r}) \\
& =\int_{\mathbf{0}}^{\mathbf{z}} B_{C,}(\mathbf{r}, \mathbf{y}) \cdot A(\mathbf{x}, \mathrm{d} \mathbf{r}) \\
& =\int_{0}^{\mathbf{x}} \int_{\mathbf{0}}^{\mathbf{z}} B_{C},(\mathbf{r}, \mathbf{y}) \cdot A_{D},(\mathbf{v}, \mathrm{d} \mathbf{r}) D(\mathrm{~d} \mathbf{v}),
\end{aligned}
$$

and the first assertion follows. The second statement is proved analogously.

Proposition 2. The operator $\stackrel{C(.)}{\star}$ is associative, that is, for copulas $A, B, C, D, E$ with dimension $(k+m),(m+$ $l),(l+i), m, l$ respectively, it holds

$$
\left(A^{\stackrel{D(.)}{\star}} B\right)^{\stackrel{E(.)}{\star}} C=A^{\stackrel{D(.)}{\star}}\left(B^{\stackrel{E(.)}{\star}} C\right)
$$

Proof. By repeated application of Lemma 4 and Fubini's theorem,

$$
\begin{aligned}
\left((A \stackrel{D(\mathbf{z})}{\star} B)^{E(\mathbf{r})} C\right)(\mathbf{x}, \mathbf{y}) & =\int_{\mathbf{0}}^{\mathbf{r}}(A \stackrel{D(z)}{\star} B)_{, E}(\mathbf{x}, \mathbf{v}) C_{E,}(\mathbf{v}, \mathbf{y}) E(\mathrm{~d} \mathbf{v}) \\
& =\int_{\mathbf{0}}^{\mathbf{r}} \int_{\mathbf{0}}^{\mathbf{z}} A_{, D}(\mathbf{x}, \xi) B_{, E}(\mathrm{~d} \xi, \mathbf{v}) C_{E,}(\mathbf{v}, \mathbf{y}) E(\mathrm{~d} \mathbf{v}) \\
& =\int_{\mathbf{0}}^{\mathbf{z}} A_{, D}(\mathbf{x}, \xi)\left(B^{E(\mathbf{r})}{ }^{\star} C\right)(\mathrm{d} \xi, \mathbf{y}) \\
& =\int_{\mathbf{0}}^{\mathbf{z}} A_{, D}(\mathbf{x}, \xi)\left(B^{\stackrel{E(\mathbf{r})}{\star}} C\right)_{D,}(\xi, \mathbf{y}) D(\mathrm{~d} \xi) \\
& =\left(A^{D(\mathbf{z})}\left(B^{E(\mathbf{r})} C\right)\right)^{*}(\mathbf{x}, \mathbf{y}) .
\end{aligned}
$$

\section{Multivariate Markov processes and copulas}

Let $\mathbf{X}=\left(\mathbf{X}_{t}\right)_{t \in \mathcal{T}}$ be a $n$-dimensional stochastic process with coordinates $X^{i}=\left(X_{t}^{i}\right)_{t \in \mathcal{T}}, i=1, \ldots, n$, defined on some filtered probability space $\left(\Omega, \mathcal{F}, \mathbb{F}=\left(\mathcal{F}_{t}\right)_{t \in \mathcal{T}}, \mathbf{P}\right)$. In this section we investigate properties of the copulas associated with $\mathbf{X}$ in case $\mathbf{X}$ is a Markov process.

We denote by $C^{s t}$ the $(n+n)$-dimensional copula between $\mathbf{X}_{s}, \mathbf{X}_{t}, s<t$,

$$
\mathbf{P}\left(X_{s}^{1} \leq x_{1}, \ldots, X_{s}^{n} \leq x_{n}, X_{t}^{1} \leq y_{1}, \ldots, X_{t}^{n} \leq y_{n}\right)=C^{s t}\left(F_{X_{s}^{1}}\left(x_{1}\right), \ldots, F_{X_{s}^{n}}\left(x_{n}\right), F_{X_{t}^{1}}\left(y_{1}\right), \ldots, F_{X_{t}^{n}}\left(y_{n}\right)\right) .
$$

We write $C^{t}$ for the copula of the vector $\mathbf{X}_{t}$. Observe that in the following all time points $s, t, \ldots$ are elements of the set $\mathcal{T}$.

For $\mathbf{x} \in \mathbb{R}^{n}$ and Borel sets $y \in \mathfrak{B}\left(\mathbb{R}^{n}\right)$ we denote

$$
\mathcal{P}(s, \mathbf{x} ; t, y)=\mathbf{P}\left(\mathbf{X}_{t} \in y \mid \mathbf{X}_{s}=\mathbf{x}\right)
$$


and call $\mathcal{P}$ transition probability function of $\mathbf{X}$. As usual we take a regular version of the conditional probability $\mathbf{P}\left(\mathbf{X}_{t} \in \mathcal{y} \mid \mathbf{X}_{s}=\mathbf{x}\right)$. Observe that $\mathbf{X}$ is not assumed to be a Markov process.

Theorem 1. The transition probability function $\mathcal{P}$ of $\mathbf{X}$ satisfies the Chapman-Kolmogorov equation,

$$
\mathcal{P}(s, \mathbf{x} ; t, y)=\int_{\mathbb{R}^{n}} \mathcal{P}(u, \mathbf{z} ; t, y) \mathcal{P}(s, \mathbf{x} ; u, d \mathbf{z}), s<u<t, y \in \mathfrak{B}\left(\mathbb{R}^{n}\right),
$$

if and only if

$$
C^{s t}=C^{s u} \stackrel{C^{u}}{\star} C^{u t} \text { for all } s<u<t
$$

Proof. Suppose that (10) holds true. It suffices to verify the Chapman-Kolmogorov equation for Borel sets $y=(-\infty, \mathbf{y})$. For $s<u<t$, by using Lemma 1 , we obtain

$$
\begin{aligned}
\int_{\mathbb{R}^{n}} \mathcal{P}(u, \mathbf{z} ; t,(-\infty, \mathbf{y})) \mathcal{P}(s, \mathbf{x} ; u, \mathrm{~d} \mathbf{z}) & =\int_{\mathbb{R}^{n}} C_{C^{u}}^{u t},\left(\mathbf{F}_{\mathbf{X}_{u}}(\mathbf{z}), \mathbf{F}_{\mathbf{X}_{t}}(\mathbf{y})\right) C_{C^{s},\left(\mathbf{F}_{\mathbf{X}_{s}}(\mathbf{x}), \mathbf{F}_{\mathbf{X}_{u}}(\mathrm{~d} \mathbf{z})\right)} \\
& =\int_{[0,1]^{n}} C_{C^{u}}^{u t},\left(\mathbf{v}, \mathbf{F}_{\mathbf{X}_{t}}(\mathbf{y})\right) C_{C^{s},}^{s u}\left(\mathbf{F}_{\mathbf{X}_{s}}(\mathbf{x}), \mathrm{d} \mathbf{v}\right) .
\end{aligned}
$$

By applying Lemma 4 and (10) we conclude,

$$
\begin{aligned}
& \int_{\mathbb{R}^{n}} \mathcal{P}(u, \mathbf{z} ; t,(-\infty, \mathbf{y})) \mathcal{P}(s, \mathbf{x} ; u, \mathrm{~d} \mathbf{z})=\left(C^{s u} \stackrel{C^{u}}{\star} C^{u t}\right)_{C^{s},}\left(\mathbf{F}_{\mathbf{X}_{s}}(\mathbf{x}), \mathbf{F}_{\mathbf{X}_{t}}(\mathbf{y})\right) \\
& =C_{C^{s}}^{s t},\left(\mathbf{F}_{\mathbf{X}_{s}}(\mathbf{x}), \mathbf{F}_{\mathbf{X}_{t}}(\mathbf{y})\right) \\
& =\mathcal{P}(s, \mathbf{x} ; t,(-\infty, \mathbf{y})) \text {. }
\end{aligned}
$$

Conversely, if the Chapman-Kolmogorov equation holds true, we get from the above calculation that

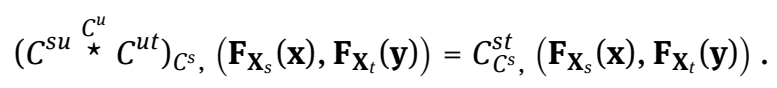

This implies (10), where we make use of our assumption that all copulas are coordinate-wise linearly interpolated for points where the copula is not uniquely defined.

Definition 3. A family of $2 n$-dimensional copulas $C^{s t}, s, t \in \mathcal{T}, s<t$, is called a Markov family if $C^{s u}(\mathbf{1}, \cdot)=$ $C^{u t}(\cdot, 1)$ for all $s<u<t$ and $(10)$ holds true.

Remark 2. If the copulas $C^{\text {st }}(\mathbf{x}, \mathbf{y})$ admit densities $c^{\text {st }}$,

$$
C^{s t}(\mathbf{x}, \mathbf{y})=\int_{\mathbf{0}}^{\mathbf{x}} \int_{\mathbf{0}}^{\mathbf{y}} c^{s t}(\mathbf{u}, \mathbf{v}) \mathrm{d} \mathbf{u} \mathrm{d} \mathbf{v}
$$

then by applying Remark 1 condition (10) translates to

$$
c^{s t}(\mathbf{u}, \mathbf{v})=\int_{[0,1]^{n}} \frac{c^{s u}(\mathbf{u}, \mathbf{z}) c^{u t}(\mathbf{z}, \mathbf{v})}{\int_{[0,1]^{n}} c^{u t}(\mathbf{z}, \mathbf{r}) \mathrm{d} \mathbf{r}} \mathrm{d} \mathbf{z}
$$

As is well-known, fulfilment the Chapman-Kolomogorov equation is sufficient but not necessary for $\mathbf{X}$ to be a Markov process. The Markov property,

$$
\mathbf{P}\left(\mathbf{X}_{t_{p}}<\mathbf{y} \mid \sigma\left(\mathbf{X}_{t_{1}}, \ldots, \mathbf{X}_{t_{p-1}}\right)\right)=\mathbf{P}\left(\mathbf{X}_{t_{p}}<\mathbf{y} \mid \sigma\left(\mathbf{X}_{t_{p-1}}\right)\right),
$$

for all $t_{1}<\cdots<t_{p-1}<t_{p}, \mathbf{y} \in \mathbb{R}^{n}$, requires further properties of the copulas related with $\mathbf{X}$, which we formulate now. Recall that the ${ }^{C(.)}{ }^{\star}$ operator is associative, that is, for repeated application of the operator the order of application does not matter. 
Theorem 2. The n-dimensional process $\mathbf{X}$ is a Markov process if and only if for all $t_{1}<t_{2}<\cdots<t_{p}$ the copula $C^{t_{1}, \ldots, t_{p}}$ of $\left(\mathbf{X}_{t_{1}}, \ldots, \mathbf{X}_{t_{p}}\right)$ satisfies

$$
C^{t_{1}, \ldots, t_{p}}=C^{t_{1}, t_{2}} \stackrel{C^{t_{2}}(.)}{\star} C^{t_{2}, t_{3}} \stackrel{C^{t_{3}}(.)}{\star} \ldots \stackrel{C^{t_{p-1}}(.)}{\star} C^{t_{p-1}, t_{p}} .
$$

Proof. First, by combining Proposition 1 and Proposition 2 the right hand side of (12) defines a copula. For the case $p=3$, the Markov property (11) is equivalent to the conditional independence of $\mathbf{X}_{t_{1}}, \mathbf{X}_{t_{3}}$ given $\mathbf{X}_{t_{2}}$. By Lemma 3 this is equivalent to (12), proving the assertion for $p=3$. For the general case we proceed by induction. Let $\mathbf{X}$ be a Markov process. The Markov property (11) implies

$$
\begin{aligned}
C^{t_{1}, \ldots, t_{p}}\left(\mathbf{F}_{\mathbf{X}_{t_{1}}}\left(\mathbf{x}_{1}\right), \ldots, \mathbf{F}_{\mathbf{X}_{t_{p}}}\left(\mathbf{x}_{p}\right)\right)=\mathbf{P}\left(\mathbf{X}_{t_{1}}<\mathbf{x}_{1}, \ldots, \mathbf{X}_{t_{p}}<\mathbf{x}_{p}\right) \\
=\int_{\mathbf{0}}^{\mathbf{x}_{p-1}} C^{t_{1}, \ldots, t_{p-1}}\left(\mathbf{F}_{\mathbf{X}_{t_{1}}}\left(\mathbf{x}_{1}\right), \ldots, \mathbf{F}_{\mathbf{X}_{t_{p-2}}}\left(\mathbf{x}_{p-2}\right), \mathbf{F}_{\mathbf{X}_{t_{p-1}}}\left(\mathrm{~d} \mathbf{z}_{p-1}\right)\right) \\
=\left(C^{t_{1}, \ldots, t_{p-1}} \stackrel{\mathbf{P}\left(\mathbf{X}_{t_{p}}<\mathbf{x}_{p} \mid \mathbf{X}_{t_{p-1}}=\mathbf{z}_{p-1}\right)}{C_{p-1}^{t_{p-1}\left(\mathbf{F}_{\mathbf{x}_{t_{p-1}}}\left(\mathbf{x}_{p-1}\right)\right)}} C^{t_{p-1}, t_{p}}\right)\left(\mathbf{F}_{\mathbf{X}_{t_{1}}}\left(\mathbf{x}_{1}\right), \ldots, \mathbf{F}_{\mathbf{X}_{t_{p-2}}}\left(\mathbf{x}_{p-2}\right), \mathbf{F}_{\mathbf{X}_{t_{p}}}\left(\mathbf{x}_{p}\right)\right) .
\end{aligned}
$$

By induction this yields (12), where we take into account that all copulas are coordinate-wise linearly interpolated for points where the copula is not uniquely defined. Now assume that (12) holds true. Then for any $q$ with $t_{1}<t_{q}<t_{p}$

$$
C^{t_{1}, \ldots, t_{p}}=C^{t_{1}, t_{2}, \ldots, t_{q}}{ }^{C_{q}^{t_{q}}(.)} C^{t_{q}, \ldots, t_{p}} .
$$

By Lemma 3 this implies that $\left(\mathbf{X}_{t_{1}}, \ldots, \mathbf{X}_{t_{q-1}}\right)$ and $\left(\mathbf{X}_{t_{q+1}}, \ldots, \mathbf{X}_{t_{p}}\right)$ are conditionally independent given $\mathbf{X}_{t_{q}}$. This proves the assertion.

Remark 3. For the bivariate case, $n=2$, discrete time and, most importantly, if the copulas $C^{s, t}(\mathbf{x}, \cdot)$ and $C^{s, t}(\cdot, \mathbf{y})$ are absolutely continuous for all $\mathbf{x}$ and $\mathbf{y}$, the result of Theorem 2 reduces to Theorem 5.1 in [10]. However, for our construction of a Markov family of copulas in Section 4 it is crucial to depart from the restriction of absolute continuity, which in turn requires a more general definition of the product operator. Theorem 5.1 in [10] is formulated in terms of a product operator that has been introduced in [13] and which is based on derivatives of copulas. For the proof of Theorem 5.1, [10] refers to [13], whose proof uses conditional distributions which are basically conditioned copulas. The proof carries over to our situation as soon as conditioned copulas are identified as derivative of a copula with respect to a copula (see Definition 1 and Lemma 1) and the product operator is generalized according to our Definition 2.

Remark 4. It is straightforward to extend our copula analysis to higher order Markov processes, following the lines in [13]. For a Markov process of order $k \geq 1$ equation (12) has to be replaced by the following condition. For $t_{1}<t_{2}<\cdots<t_{p}, p \geq k+1$, the copula $C^{t_{1}, \ldots, t_{p}}$ of $\left(\mathbf{X}_{t_{1}}, \ldots, \mathbf{X}_{t_{p}}\right)$ satisfies

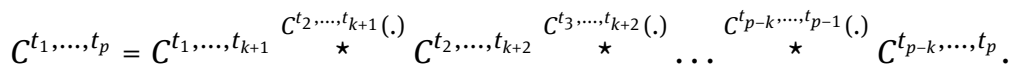

Remark 5. Given a Markov family $C^{s t}, s, t \in \mathcal{T}, s<t$, we can define finite dimensional copulas $C^{t_{1}, \ldots, t_{p}}$ by (12) and combine these with an arbitrarily specified flow $\mathbf{F}_{t}(\mathbf{x})=\left(F_{X_{t}^{1}}\left(x_{1}\right), \ldots, F_{X_{t}^{n}}\left(x_{n}\right)\right)$ of marginal one-dimensional distributions $F_{X_{t}^{i}}$ to obtain finite dimensional distributions

$$
\mathbf{P}\left(\mathbf{X}_{t_{1}}<\mathbf{x}_{1}, \ldots, \mathbf{X}_{t_{p}}<\mathbf{x}_{p}\right)=C^{t_{1}, \ldots, t_{p}}\left(\mathbf{F}_{t_{1}}\left(\mathbf{x}_{1}\right), \ldots, \mathbf{F}_{t_{p}}\left(\mathbf{x}_{p}\right)\right) .
$$

By applying Kolomogorov's construction theorem for stochastic processes there exists a Markov process $\mathbf{X}$ with the given copulas and marginal distributions. 
Starting with a known Markov process with associated Markov family $C^{\text {st }}$ one can construct new Markov processes $\mathbf{X}$ by means of the same Markov family but with a modified flow of univariate marginal distributions. Observe, that in general, it is not possible to modify the flow of joint distributions $\mathbf{P}\left(X_{t}^{1}<x_{1}, \ldots, X_{t}^{n}<x_{n}\right)$ because they involve the "spatial" copulas $C^{t}$.

To give an example, consider a 2-dimensional Wiener process with instantaneous correlation $\rho$. The associated Markov family of copulas is just the Gaussian copula

$$
C^{s t}(\mathbf{x}, \mathbf{y})=\mathrm{N}_{4}\left(\mathrm{~N}^{(-1)}\left(x_{1}\right), \mathrm{N}^{(-1)}\left(x_{2}\right), \mathrm{N}^{(-1)}\left(y_{1}\right), \mathrm{N}^{(-1)}\left(y_{2}\right) ; \Sigma\right),
$$

where $\mathrm{N}^{(-1)}$ is the inverse of the standard normal distribution and $\mathrm{N}_{4}$ is the distribution function of a 4dimensional standard normal distribution with correlation matrix

$$
\Sigma=\left(\begin{array}{cccc}
1 & \rho & \sqrt{\frac{s}{t}} & \rho \sqrt{\frac{s}{t}} \\
\rho & 1 & \rho \sqrt{\frac{s}{t}} & \sqrt{\frac{s}{t}} \\
\sqrt{\frac{s}{t}} & \rho \sqrt{\frac{s}{t}} & 1 & \rho \\
\rho \sqrt{\frac{s}{t}} & \sqrt{\frac{s}{t}} & \rho & 1
\end{array}\right) .
$$

By construction the family $C^{\text {st }}$ is a Markov family that can be used to construct a 2-dimensional Markov process, which is non-Gaussian but admits the dependence structure of a multivariate Wiener process.

It would be interesting to study other elliptic copulas or Frank and Clayton copulas for the temporal dependence of a processes and give conditions for the Markov property. However, this seems still a challenge. The main reason is that most continuous time Markov process are a kind of jump diffusions whose discrete time distributions look mainly like normal or poisson distributions. [5] considers diffusion type processes or jump-diffusions and give conditions on the diffusion coefficients and the jump-measure to ensure the Markov property. However, to find continuous time Markov processes with a temporary dependence based on elliptic distributions, like the t-distribution is still a challenge.

\section{A Fréchet-type copula family that satisfies the Chapman-Kolmogorov equation}

We provide a recipe for constructing a wide sample class of Markov families $C^{s t}, s, t \in \mathcal{T}, s<t$. Given a Markov family $C^{s t}, s, t \in \mathcal{T}, s<t$, one can construct a corresponding Markov process $\mathbf{X}$ as outlined in Remark 5 . We construct Markov families by convex combination of "extreme" copulas. This construction can be seen as a generalization of the copula family in [11], Example 4.5, to the multivariate case.

Let $C$ be a given $n$-dimensional copula, which will serve as "stationary spatial" copula, i.e., the copula of the random vector $\mathbf{X}_{t}$ is $C^{t}=C$, for all $t \in \mathcal{T}$. Recall that the marginal distributions of $X_{t}^{i}$ are still arbitrary because they do not enter condition (10) for the Chapman-Kolomogorov equation to hold true. We define the following "extreme" copula $M$ and the product copula

$$
\begin{aligned}
M(\mathbf{x}, \mathbf{y}) & =C(\min (\mathbf{x}, \mathbf{y})) \\
P(\mathbf{x}, \mathbf{y}) & =C(\mathbf{x}) C(\mathbf{y}),
\end{aligned}
$$

where the minimum is understood coordinate-wise. To see that $M$ is indeed a copula, let $\mathbf{X}$ be a $n$-dimensional random vector with distribution function $C$. Then

$$
M(\mathbf{x}, \mathbf{y})=\mathbf{P}(\mathbf{X}<\mathbf{x}, \mathbf{X}<\mathbf{y}), \mathbf{x}, \mathbf{y} \in[0,1]^{n},
$$

which shows that $M$ is a distribution function on $[0,1]^{2 n}$. That $M$ has uniform margins is obvious. This also shows that $M$ is a kind of total dependence copula. Further $P$ is a kind of independence copula, it is the multivariate distribution of $(\mathbf{X}, \mathbf{Y})$ with $\mathbf{X}$ and $\mathbf{Y}$ independent and distributed according to $C$. Clearly $P$ has uniform margins.

The following properties are crucial for our construction of a Markov family of copulas below. 
Lemma 5. Let $K(\mathbf{x}, \mathbf{y})$ be a $2 n$-dimensional copula with $K(\mathbf{x}, \mathbf{1})=C(\mathbf{x}), K(\mathbf{1}, \mathbf{y})=C(\mathbf{y})$. Then

$$
\begin{gathered}
\stackrel{C}{{ }^{\star}} P={ }^{\stackrel{C}{\star}} K=P \\
K^{\circledR}{ }^{\star} M=M^{\star}{ }^{\star} K=K .
\end{gathered}
$$

Proof. We show that $P$ acts as a null element with respect to the ${ }^{\circledR}$ product. We have $P, C(\mathbf{x}, \mathbf{y})=C(\mathbf{x})$ and $P_{C},(\mathbf{x}, \mathbf{y})=C(\mathbf{y})$. This yields

$$
\begin{aligned}
K^{\star} P(\mathbf{x}, \mathbf{y}) & =\int_{\mathbf{0}}^{\mathbf{1}} K_{, C}(\mathbf{x}, \mathbf{z}) P_{C,}(\mathbf{z}, \mathbf{y}) C(\mathrm{~d} \mathbf{z}) \\
& =C(\mathbf{y}) \int_{\mathbf{0}}^{\mathbf{1}} K_{, C}(\mathbf{x}, \mathbf{z}) C(\mathrm{~d} \mathbf{z})=C(\mathbf{y}) K(\mathbf{x}, \mathbf{1})=C(\mathbf{y}) C(\mathbf{x}) \\
& =P(\mathbf{x}, \mathbf{y}) .
\end{aligned}
$$

The same arguments apply for the null property with the left hand side ${ }^{\star}$ multiplication by $P$. This proves (13). The copula $M$ is the identity with respect to the ${ }^{\complement}{ }^{C}$ operator. To verify this, the derivatives of $M$ with respect to $C$ are obviously $M_{, C}(\mathbf{x}, \mathbf{z})=\mathbf{1}_{\{\mathbf{z} \leq \mathbf{x}\}}$ and $\left.M_{C}, \mathbf{z}, \mathbf{y}\right)=\mathbf{1}_{\{\mathbf{z} \leq \mathbf{y}\}}$. This implies as requested

$$
\begin{aligned}
K^{\star}{ }^{C} M(\mathbf{x}, \mathbf{y}) & =\int_{\mathbf{0}}^{\mathbf{1}} K_{, C}(\mathbf{x}, \mathbf{z}) M_{C,}(\mathbf{z}, \mathbf{y}) C(\mathrm{~d} \mathbf{z}) \\
& =\int_{\mathbf{0}}^{\mathbf{1}} K_{, C}(\mathbf{x}, \mathbf{z}) \mathbf{1}_{\{\mathbf{z} \leq \mathbf{y}\}} C(\mathrm{~d} \mathbf{z}) \\
& =K(\mathbf{x}, \mathbf{y}) .
\end{aligned}
$$

Again, the left side identity property is shown analogously.

The third building block for our convex combinations of copulas will be an appropriately chosen multivariate copula replacement for the lower Fréchet-Hoeffding bound, which is $W(x, y)=\max (x+y-1,0)$ for dimension two. As is well-known, for higher dimension the lower Fréchet-Hoeffding bound is in general not a copula. We proceed as follows. Let $\mathbf{X}$ be a $n$-dimensional uniform random vector whose distribution function is our copula $C$ above. Suppose there exists a bijective mapping $T:[0,1]^{n} \rightarrow[0,1]^{n}$ with the following properties

$$
\begin{aligned}
\mathbf{P}(T(\mathbf{X})<\mathbf{x}) & =C(\mathbf{x}) \\
T^{2}(\mathbf{x}) & =\mathbf{x} \\
C(\{\mathbf{x}: T(\mathbf{x}) \neq \mathbf{x}\}) & >0 .
\end{aligned}
$$

An example is the mapping $T(\mathbf{x})=\mathbf{1}-\mathbf{x}$ in case the copula $C$ is symmetric,

$$
C(\mathbf{x})=\hat{C}(\mathbf{1}-\mathbf{x}),
$$

with $\hat{C}$ the survival copula associated with $C$.

Now define the $2 n$-dimensional copula $W$ by

$$
W(\mathbf{x}, \mathbf{y})=\mathbf{P}(\mathbf{X}<\mathbf{x}, T(\mathbf{X})<\mathbf{y}), \mathbf{x}, \mathbf{y} \in[0,1]^{n} .
$$

Lemma 6. We have

$$
W^{\star} \stackrel{C}{*} W=M
$$


Proof. Since

$$
\begin{aligned}
W(\mathbf{x}, \mathbf{y}) & =\mathbf{P}(\mathbf{X}<\mathbf{x}, T(\mathbf{X})<\mathbf{y}) \\
& =\int_{\mathbf{0}}^{\mathbf{x}} \mathbf{P}(T(\mathbf{X})<\mathbf{y} \mid \mathbf{X}=\mathbf{z}) C(\mathrm{~d} \mathbf{z}) \\
& =\int_{\mathbf{0}}^{\mathbf{x}} \mathbf{1}_{\{T(\mathbf{z})<\mathbf{y}\}} C(\mathrm{~d} \mathbf{z})
\end{aligned}
$$

we have

$$
W_{C,}(\mathbf{z}, \mathbf{y})=\mathbf{1}_{\{T(\mathbf{z})<\mathbf{y}\}} \cdot
$$

Similarly

$$
W_{, C}(\mathbf{x}, \mathbf{z})=\mathbf{1}_{\{T(\mathbf{z})<\mathbf{x}\}} \cdot
$$

Finally,

$$
\begin{aligned}
{ }_{W}{ }^{C} W(\mathbf{x}, \mathbf{y}) & =\int_{\mathbf{0}}^{1} \mathbf{1}_{\{T(\mathbf{z})<\mathbf{x}\}} \mathbf{1}_{\{T(\mathbf{z})<\mathbf{y}\}} C(\mathrm{~d} \mathbf{z}) \\
& =\mathbf{P}(\mathbf{X}<\min (\mathbf{x}, \mathbf{y})) \\
& =M(\mathbf{x}, \mathbf{y}) .
\end{aligned}
$$

We attempt to construct a Markov family $C^{s t}, s, t \in \mathcal{T}, s<t$, by convex combination of the copulas $P, M, W$,

$$
C^{s t}=\alpha(s, t) W+(1-\alpha(s, t)-\beta(s, t)) P+\beta(s, t) M,
$$

with $\alpha(s, t), \beta(s, t) \geq 0, \alpha(s, t)+\beta(s, t) \leq 1$.

As in [11], Example 4.5, we obtain the following result.

Proposition 3. The copula family $C^{s t}, s, t \in \mathcal{T}, s<t$, is a Markov family if and only if

$$
\begin{aligned}
& \alpha(s, t)=\beta(s, u) \alpha(u, t)+\alpha(s, u) \beta(u, t) \\
& \beta(s, t)=\alpha(s, u) \alpha(u, t)+\beta(s, u) \beta(u, t) .
\end{aligned}
$$

Proof. First $C^{u}=C$ by construction. Then for $s<u<t$, using Lemmas 5 and 6 we have

$$
\begin{aligned}
C^{s u}{ }_{\star}^{C^{u}} C^{u t}= & (\alpha(s, u) W+(1-\alpha(s, u)-\beta(s, u)) P+\beta(s, u) M) \\
C^{u} & (\alpha(u, t) W+(1-\alpha(u, t)-\beta(u, t)) P+\beta(u, t) M) \\
= & \alpha(s, u) \alpha(u, t) M+\alpha(s, u)(1-\alpha(u, t)-\beta(u, t)) P+\alpha(s, u) \beta(u, t) W \\
& +(1-\alpha(s, u)-\beta(s, u)) \alpha(u, t) P \\
& +(1-\alpha(s, u)-\beta(s, u))(1-\alpha(u, t)-\beta(u, t)) P \\
& +(1-\alpha(s, u)-\beta(s, u)) \beta(u, t) P+\beta(s, u) \alpha(u, t) W \\
& +\beta(s, u)(1-\alpha(u, t)-\beta(u, t)) P+\beta(s, u) \beta(u, t) M .
\end{aligned}
$$

Then $C^{s u} \stackrel{C^{u}}{\star} C^{u t}=C^{s t}$ holds true if and only if (20), (21) are satisfied.

There are various solutions to the system (20), (21). We refer to [11] for examples.

An example of a homogeneous Markov family is obtained by setting

$$
\begin{aligned}
& \alpha(s, t)=a(t-s), \text { with } a(h)=\frac{\mathbf{e}^{-2 h}\left(1-\mathbf{e}^{-h}\right)}{2} \\
& \beta(s, t)=b(t-s), \text { with } b(h)=\frac{\mathbf{e}^{-2 h}\left(1+\mathbf{e}^{-h}\right)}{2},
\end{aligned}
$$


for $s<t, h>0$.

Next we calculate the infinitesimal generator associated with this example.

Let $\mathbf{X}$ be the Markov process with the Markov family $C^{s t}, s, t \in \mathcal{T}, s<t$, constructed by (19) with conditions (20), (21). For simplicity assume that $\mathbf{X}$ has uniform marginal distributions, $F_{X_{t}^{i}}(x)=x$. Let $g:[0,1]^{n} \rightarrow$ $\mathbb{R}$ be a bounded Borel measurable function. Recall the following derivatives of the building blocks of $C^{s t}$,

$$
W_{C,}(\mathbf{x}, \mathbf{y})=\mathbf{1}_{\{T(\mathbf{x})<\mathbf{y}\}}, P_{C},(\mathbf{x}, \mathbf{y})=C(\mathbf{y}), M_{C,}(\mathbf{x}, \mathbf{y})=\mathbf{1}_{\{\mathbf{x}<\mathbf{y}\}} .
$$

Then

$$
\int_{[0,1]^{n}} g(\mathbf{y}) \mathbf{P}\left(\mathbf{X}_{t} \in \mathrm{d} \mathbf{y} \mid \mathbf{X}_{s}=\mathbf{x}\right)=\alpha(s, t) g(T(\mathbf{x}))+(1-\alpha(s, t)-\beta(s, t)) \int_{[0,1]^{n}} g(\mathbf{y}) C(\mathrm{~d} \mathbf{y})+\beta(s, t) g(\mathbf{x}) .
$$

The behaviour of the process $\mathbf{X}$ can be interpreted as the following jump-process. From $\mathbf{X}_{s}=\mathbf{x}$ at time $s$ to time $t$ the process either stays at $\mathbf{x}$ with probability $\beta(s, t)$, or it jumps from $\mathbf{x}$ to $T(\mathbf{x})$ with probability $\alpha(s, t)$, or, with probability $(1-\alpha(s, t)-\beta(s, t))$ it jumps to a new position $\mathbf{y}$ with distribution $C(\mathrm{~d} \mathbf{y})$ independently from the current position $\mathbf{x}$.

In case of the homogeneous Markov family of copulas (22), (23) it is straightforward to determine the infinitesimal generator of $\mathbf{X}$. For $h=(t-s)>0$,

$$
\begin{aligned}
\frac{\int_{[0,1]^{n}} g(\mathbf{y}) P\left(\mathbf{X}_{t} \in \mathrm{d} \mathbf{y} \mid \mathbf{X}_{s}=\mathbf{x}\right)-g(\mathbf{x})}{h}= & \frac{\mathbf{e}^{-2 h}\left(1-\mathbf{e}^{h}\right)}{2 h}(g(T(\mathbf{x}))-g(\mathbf{x}))+\frac{1-\mathbf{e}^{-2 h}}{h}\left(\int_{[0,1]^{n}} g(\mathbf{y}) C(\mathrm{~d} \mathbf{y})-g(\mathbf{x})\right) \\
& +\frac{\mathbf{e}^{-2 h}\left(1+\mathbf{e}^{h}\right)}{2 h}(g(\mathbf{x})-g(\mathbf{x})) .
\end{aligned}
$$

As $h$ tends to 0 we get the infinitesimal generator $\mathcal{A}$ as the limit

$$
\mathcal{A} g(\mathbf{x})=k_{1} g(\mathbf{x})+k_{2} g(T(\mathbf{x}))+k_{3} \int_{[0,1]^{n}} g(\mathbf{y}) C(\mathrm{~d} \mathbf{y})
$$

with constants $k_{1}, k_{2}, k_{3}$.

\section{References}

[1] Abegaz, F. and Naik-Nimbalkar, U. (2008). Modeling statistical dependence of Markov chains via copula models. J. Statist. Plann. Inference, 138(4), 1131-1146.

[2] Beare, B.K. (2010). Copulas and temporal dependence. Econometrica, 78(1), 395-410.

[3] Beare, B.K. and Seo, J. (2015) Vine copula specification for stationary multivariate Markov chains. J. Time Series Anal., 36, 228-246.

[4] Bielecki, T. R., Crépey, S., Jeanblanc, M., and Zargari, B. (2012). Valuation and hedging of CDS counterparty exposure in a Markov copula model. Int. J. Theor. Appl. Finance, 15(01).

[5] Bielecki, T. R., Jakubowski, J., Vidozzi, A., and Vidozzi, L. (2008). Study of dependence for some stochastic processes. Stoch. Anal. Appl., 26(4), 903-924.

[6] Brechmann, E.C., Czado, C., and Aas, K. (2012) Truncated regular vines in high dimensions with application to financial data. Canad. J. Statist., 40 (1), 68-85.

[7] Chen, X. and Fan, Y. (2006) Estimation of copula-based semiparametric time series models. J. Econometrics, 130, $307-335$.

[8] Chen, X. , Wu, W.B., and Yi, Y. (2009) Efficient estimation of copula-based semiparametric Markov models. Ann. Statist., 37 (6B), 4214-4253.

[9] Cherubini, U., Mulinacci, S., Gobbi, F., and Romagnoli, S. (2011a). Dynamic Copula Methods in Finance. Wiley.

[10] Cherubini, U., Mulinacci, S., and Romagnoli, S. (2011b). A copula-based model of speculative price dynamics in discrete time. J. Multivariate Anal., 102(6), 1047-1063.

[11] Darsow, W., Nguyen, B., and Olsen, E. (1992). Copulas and Markov processes. Illinois J. Math, 36(4), 600-642.

[12] Haff, I.H. (2012) Estimating the parameters of a pair copula construction. Bernoulli, 19(2), 462-491.

[13] Ibragimov, R. (2009). Copula-based characterizations for higher order Markov processes. Econometric Theory, 25(03), 819-846. 
[14] Joe, H. (1997). Multivariate Models and Dependence Concepts. Chapman \& Hall/CRC.

[15] McNeil, A.J., Frey, R., and Embrechts, P. (2010). Quantitative Risk Management: Concepts, Techniques, and Tools. Princeton University Press.

[16] Nelsen, R. (1999). An Introduction to Copulas. Springer Verlag, New York.

[17] Rémillard, B., Papageorgiou, N., and Soustra, F. (2012) Copula-based semiparametric models for multivariate time series. J. Multivariate Anal., 110, 30-42.

[18] Simard, C. and Rémillard, B. (2015) Forecasting time series with multivariate copulas. Depend. Model., 3, 59-82.

[19] Stöber, J. and Czado, C. (2014) Regime switches in the dependence structure of multidimensional financial data. Comput. Stat. Data An., 76, 672-686.

[20] Yi, W. and Liao, S.S. (2010) Statistical properties of parametric estimators for Markov chain vectors based on copula models. J. Statist. Plann. Inference, 140, 1465-1480. 